\title{
Anticrab cavities for the removal of spurious vertical bunch rotations caused by crab cavities
}

\author{
G. Burt, ${ }^{1}$ A. Latina, ${ }^{2}$ D. Schulte, ${ }^{2}$ A. C. Dexter, ${ }^{1}$ and P. A. McIntosh ${ }^{3}$ \\ ${ }^{1}$ Cockcroft Institute, Lancaster University, Lancaster, LA1 4YR, United Kingdom \\ ${ }^{2}$ CERN, CH-1211 Geneva 23, Switzerland \\ ${ }^{3}$ STFC, Daresbury Laboratories, Warrington, WA4 4AD, United Kingdom \\ (Received 8 April 2008; published 26 September 2008)
}

\begin{abstract}
Many particle accelerators are proposing the use of crab cavities to correct for accelerator crossing angles or for the production of short bunches in light sources. These cavities produce a rotation to the bunch in a well-defined polarization plane. If the plane of the rotation does not align with the horizontal axis of the accelerator, the bunch will receive a small amount of spurious vertical bunch rotation. For accelerators with small vertical beam sizes and large beam-beam effects, this can cause significant unwanted effects. In this paper we propose the use of a 2 nd smaller crab cavity in the vertical plane in order to cancel this effect and investigate its use in numerical simulations.
\end{abstract}

DOI: 10.1103/PhysRevSTAB.11.092801

PACS numbers: 29.20.Ej, 41.75.Ht, 41.85.-p

\section{INTRODUCTION}

Particle colliders often employ a finite crossing angle between colliding bunches. The associated loss of luminosity for long thin bunches can be recovered by rotating the bunches prior to collision, such that the bunches will now have an effective head-on collision. One method of rotating the bunches is to use a crab cavity [1]. These devices are deflecting mode cavities in which the cavity phase is set such that the head and tail of the bunch receive equal and opposite kicks, and the center of mass receives zero kick. This will impart a transverse momentum to the bunch which causes the bunch to rotate as it travels along the beam line. Crab cavities are required for several particle colliders such as KEK-B [2], the International Linear Collider (ILC) [3], the LHC upgrade [4], and CLIC [5], and also a number of light sources such as the Argonne light source, ALS [6].

Crab cavities typically operate using the $\mathrm{TM}_{110}$ mode of an rf cavity, in which the transverse electric and magnetic fields act together to kick the bunches in the same plane. This mode has a well-defined polarization in which it provides the kick. However, if the polarization of the operating mode of these crab cavities is not perfectly aligned with the horizontal axis of the accelerator, then a small amount of the crabbing effect will take place in the beams' vertical plane. This will cause the bunches to collide with a small vertical crossing angle. If the bunch has a smaller vertical beam size than horizontal beam size, this could cause a large luminosity reduction as is the case in the ILC and CLIC. Such a vertical rotation has been observed during operation at KEK-B [2]. There are also a number of other sources of vertical crabbing due to $x-y$ coupling. There are a number of approaches to correct for this vertical rotation, such as active mechanical tuners to rotate the cavity in situ, beam tuning knobs, or using correction cavities. In this paper we propose the use of vertical crab cavities, referred to as anticrab cavities, to counteract this effect. In such a scheme a smaller (singlecell) crab cavity is placed close to the crab cavity, with its operating polarization in the vertical plane. The anticrab cavity fields would be in-phase with the crab-cavity fields such that the center of mass does not receive a transverse kick in either plane. The anticrab cavity would then provide additional vertical momentum to the bunch such that the head and tail of the bunch receive equal and opposite kicks. The amplitude in the vertical can then be adjusted until the anticrab cavity cancels any vertical momentum imparted to the bunch by the crab cavity. This can be achieved in a linear collider by measuring the luminosity while varying the cavity amplitude.

In this paper we look at the theory of such a device and numerically model an anticrab cavity for use in the ILC. We then simulate the use of this anticrab cavity in the beam delivery system of the ILC, using the particle tracking code PLACET [7] together with the beam-beam code GUINEA-PIG [8].

\section{THEORY}

Using a simple geometric argument [9], which neglects beam-beam effects, for beams of bunches intersecting in a horizontal plane at a small crossing angle $\theta_{c}$, one predicts that the luminosity is reduced with respect to a head-on collision by a factor $S$ given as

$$
S=\frac{1}{\sqrt{1+\left(\frac{\sigma_{z} \theta_{c}}{2 \sigma_{z}}\right)^{2}}},
$$

where $\sigma_{z}$ and $\sigma_{x}$ are the longitudinal and horizontal Gaussian beam sizes and $\theta_{c}$ is the angle between the two colliding bunches.

In this section we assume that a crab cavity is used to eliminate this luminosity reduction and concern ourselves about additional luminosity loss when its alignment is imperfect. A crab cavity has a time varying transverse 
electric and magnetic field such that a particle traversing the cavity will experience a transverse kick given as

$$
V_{\perp}=V_{0} \sin (\omega s / c),
$$

where $s$ is the position along the bunch and the center of the bunch is given at $s=0 . V_{0}$ is the peak transverse voltage and $V_{\perp}$ is the transverse voltage experienced by the particles. The direction of $V_{\perp}$ is assumed not to be perfectly in the horizontal plane and will have components $V_{x}$ and $V_{y}$. The phase of the crab cavity is adjusted so that the head and tail experience equal and opposite forces while traversing the cavity. The effect of the cavity is to start the bunch rotating about its center. The transverse position and transverse velocity of a particle at position $s$ can be given by the linear transformation matrix, $R$, and the positions and transverse velocity at an initial position $s_{0}$ :

$$
\left[\begin{array}{c}
x \\
x^{\prime} \\
y \\
y^{\prime}
\end{array}\right] s=[R]\left[\begin{array}{c}
x \\
x^{\prime} \\
y \\
y^{\prime}
\end{array}\right]_{s_{0}} .
$$

In the ILC final focus, the focusing is very strong such that the effects of any offsets at the cavity are canceled out. However, the effects of transverse momentum are not canceled and, hence, the transverse offset of any particle in the bunch at the IP, $\Delta x$ and $\Delta y$, is given by

$$
\Delta x=R_{12} \frac{V_{x}}{E}+R_{14} \frac{V_{y}}{E} \quad \Delta y=R_{34} \frac{V_{y}}{E}+R_{32} \frac{V_{x}}{E},
$$

where $R_{m n}$ are the transfer matrix elements which relate the angular direction of the particle at the cavity to the offset of the particle at the IP [10]. If we assume $R_{14}$ and $R_{32}$ are negligible, then substituting (2) into (3b), the offset as a function of position is given as

$$
\Delta x(s)=R_{12} \frac{V_{0 x} \sin (\omega s / c)}{E} .
$$

By consideration of a particle at the front of the bunch at position $s=\sigma_{z}$, one determines bunch rotation as

$$
\theta_{\text {crab }}=\frac{\Delta x\left(\sigma_{z}\right)}{\sigma_{z}} \approx \frac{\omega}{c} R_{12} \frac{V_{0 x}}{E},
$$

where the required rotation is $\theta_{\text {crab }}=\theta_{c} / 2$.

Several factors reduce the amount of luminosity recovered using crab cavities. Phase or amplitude variations can cause a spurious center of mass kick or change the angle of rotation, respectively [11]. Wakefields induced by the charged bunch in the cavity can impart a transverse kick to following bunches [12]. In addition, if the cavity kick has a small miss alignment angle $\phi$ from the horizontal plane, then the desired horizontal crabbing rotation will be given by

$$
\theta_{\text {crab }}=\frac{\omega}{c} R_{12} \frac{V_{0}}{E} \cos (\phi)
$$

and an unwanted vertical rotation will be given by

$$
\theta_{\text {vertical }}=\frac{\omega}{c} R_{34} \frac{V_{0}}{E} \sin (\phi)=\theta_{\text {crab }} \frac{R_{34}}{R_{12}} \frac{\sin (\phi)}{\cos (\phi)} .
$$

In this situation, while the luminosity loss from horizontal rotation is still eliminated to first order, there is an additional luminosity loss from the vertical rotation determined from (1) as

$$
S=\frac{1}{\sqrt{1+\left(\frac{\sigma_{z} \theta_{\text {vertical }}}{2 \sigma_{y}}\right)^{2}}} .
$$

If the misalignment is small then, by inserting (7) into (8), the luminosity loss is determined from

$$
S=\frac{1}{\sqrt{1+\left(\frac{\sigma_{z} \theta_{\text {crab }} R_{34} \sin (\phi)}{2 \sigma_{y} R_{12}}\right)^{2}}} .
$$

If the vertical beam size is much smaller than the bunch length this can lead to a significant loss in luminosity. For example, as we will see in the next section the ILC has beam parameters such that the cavity would require to be aligned to better than $7 \mathrm{mrad}$ to keep the luminosity loss below $2 \%$. This is a tighter tolerance that is achievable when mounting the cavity; hence, some active method must be employed to rotate the cavities polarization. This can be achieved through physically rotating the cavity via a roll tuner, beam based feedback or to use a second cavity. In this paper we will consider the use of a second cavity to rotate the crabbing polarization. If a second crab cavity is placed in the beam line with a vertical crabbing effect, rather than a horizontal one, this can be used to provide an opposing kick, canceling the spurious rotation. Such a cavity would require a voltage of

$$
V_{\text {anticrab }}=V_{\text {crab }} \sin \left(\varphi_{\text {max }}\right)
$$

which would allow a single-cell anticrab cavity to compensate for the roll misalignments of several crab cavities.

Such a cavity would be susceptible to a tight phase requirement as in the regular crab cavity. The offset at the IP due to a phase error is given by

$$
\Delta y_{\mathrm{ip}}=R_{34} \frac{V_{y}}{E_{o}} \sin (\Delta \varphi) \approx R_{34} \frac{V_{y}}{E_{o}} \Delta \varphi .
$$

If we assume that we can tolerate a vertical deflection of $\sigma_{y} / 4$, then the phase tolerance is

$$
\Delta \varphi \approx \frac{E_{o} \Delta y_{\text {ip }}}{R_{34} V_{y}} .
$$

This places a limit on the maximum operating voltage of the anticrab cavity in order to avoid excessive luminosity reduction due to phase errors.

Vertical crossing angles can also be created or corrected using $x-y$ coupling. Such a system is employed at KEK-B using sextupole magnets. However, for linear colliders 
such as ILC and CLIC, where higher-order corrections are used, the implementation of an additional variable sextupole magnet between the crab cavity and the IP is likely to cause the beam size to blow up.

Alternatively it is possible to create a crossing angle with the use of the transverse velocity of the bunch, where the bunch approaches the IP at an angle, which we will refer to as an angled crossing. In this case the vertical crossing angle, excluding $x-y$ coupling, is given as

$$
\theta_{\text {vertical }}=y^{\prime}=R_{44} y^{\prime}+R_{43} y .
$$

Crossing angles of this type are often created by wakefields in linear accelerators. This is removed using angle IP feedback where a upstream kicker is used to alter $x^{\prime}$ and $y^{\prime}$ at the IP. However, there is an important differentiation between the two types of crossing angle in the mean trajectory taken by the bunch. In the case of a crab crossing the mean $y^{\prime}$ of the bunch is zero; in an angled crossing the mean $y^{\prime}$ is the crossing angle. This means that an angled crossing will only recover the lost luminosity if both bunches have equal transverse momentum. In the case of a vertical crossing angle caused by a misaligned crab cavity, correcting for this crossing angle with angle IP feedback would give one bunch a larger mean $y^{\prime}$ hence presenting a larger vertical beam size for collision. This increased beam size means that angle IP feedback cannot be used to correct for this type of crossing angle.

\section{ANTICRAB CAVITY OPERATION IN THE ILC}

The ILC has a crab crossing of $14 \mathrm{mrad}$ and collides electron and positron beams at $500 \mathrm{GeV}$ c.m. The ILC beam delivery system includes two 9 cell superconducting crab cavities. These cavities operate at a frequency of $3.9 \mathrm{GHz}$ and a maximum gradient of $5 \mathrm{MV} / \mathrm{m}$. The cavity, shown in Fig. 1, has a cell length of $38.4 \mathrm{~mm}$, an iris radius of $15 \mathrm{~mm}$ in the mid cells and $18 \mathrm{~mm}$ in the end cells, and an equator radius of $47.18 \mathrm{~mm}$.

In the ILC beam delivery system (BDS) beam line, shown in Fig. 2, the crab cavities are positioned between 13.4 and 17.4 meters from the IP. At the position of the crab cavities $R_{12}=16.4 \mathrm{~m} / \mathrm{rad}$ and $R_{34}=2.4 \mathrm{~m} / \mathrm{rad}$. At the IP

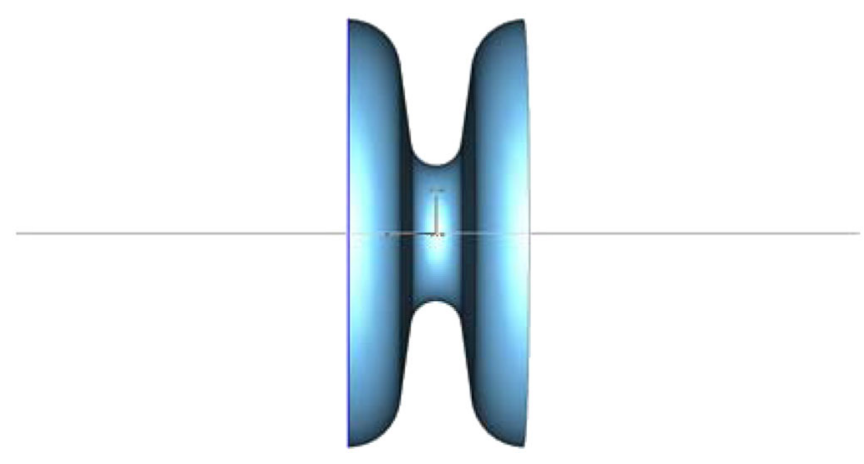

FIG. 1. (Color) The ILC crab-cavity midcell shape.

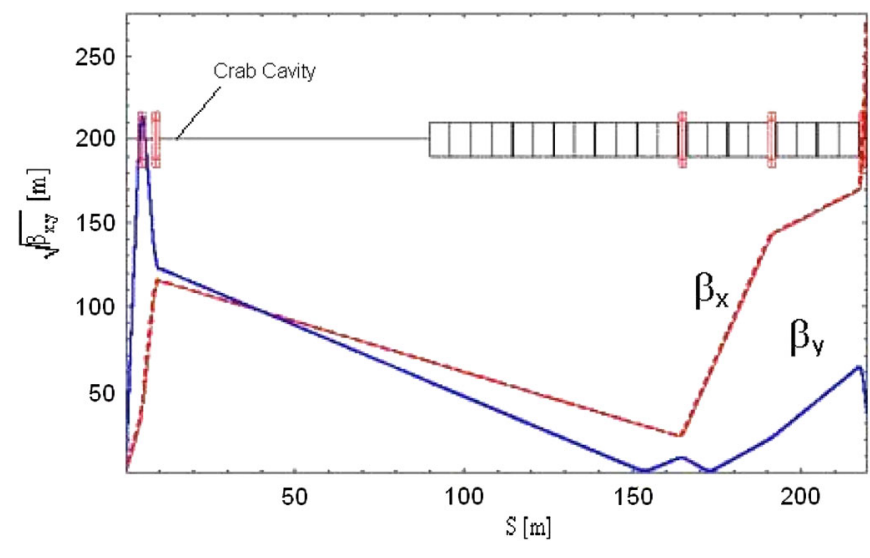

FIG. 2. (Color) The ILC BDS lattice showing the beta functions.

the bunches are focused to $\sigma_{x}=655 \mathrm{~nm}$ and $\sigma_{y}=5.7 \mathrm{~nm}$ and the bunch length is $\sigma_{z}=300 \mu \mathrm{m}$. The bunch charge is $3.2 \mathrm{nC}$ and the machine operates with $5 \mathrm{~Hz}$ train repetition frequency and 2820 bunches per train with $\sim 308 \mathrm{~ns}$ between each bunch. For these parameters, (9) shows the cavity will be required to be aligned to better than $7 \mathrm{mrad}$ to keep the luminosity loss below $2 \%$.

The ILC crab cavity has a phase tolerance of 100 millidegrees, assuming a similar tolerance we could have a gradient of up to $2 \mathrm{MV} / \mathrm{m}$ for a maximum luminosity loss of $2 \%$ from (12). Using (10) we can show that a single-cell anticrab cavity will provide enough voltage to cancel the effect of roll misalignments of up to $65 \mathrm{mrad} \sim$ 3.72 degrees in the ILC crab cavities.

MICROWAVE STUDIO has been used to model a single-cell anticrab cavity. The cavity has an equator radius of $47.6 \mathrm{~mm}$ with two $1.5 \mathrm{~mm}$ indentations on either side in one polarization to lift the degeneracy of the dipole modes. The indentations cause a $10 \mathrm{MHz}$ frequency shift between the two polarizations of the 1st dipole mode. The cavity has a large iris radius, $18 \mathrm{~mm}$, to allow the higher order modes to be strongly damped.

The dipole mode of this cavity has an $R / Q$ of $13.6 \Omega / \mathrm{cm}^{2}$ as does the same-order mode. At a gradient of $2 \mathrm{MV} / \mathrm{m}$ the peak electric field is $7.76 \mathrm{MV} / \mathrm{m}$ and the peak magnetic flux density is $58.4 \mathrm{mT}$. This cavity has a geometry factor, $G$, of $193 \Omega$. As the cavity is likely to be operating at low gradients, this cavity could possibly operate normal conducting as it will have low thermal loading at the required $0.5 \%$ duty factor.

The wakefields in this cavity are likely to be negligible in comparison to the crab cavity as it is only a single cell and will have loaded $Q$ 's at least an order of magnitude lower if superconducting and much lower if normal conducting.

\section{TRACKING AND BEAM-BEAM SIMULATIONS}

In order to verify that the anticrab cavity corrected for rotational misalignments, a series of simulations of the ILC 
beam delivery system (BDS) were performed. This was verified by integrating the particle tracking code PLACET into the beam-beam code GUINEA-PIG, which was used to calculate the luminosity of the colliding bunches of electrons and positrons.

The program PLACET (program for linear accelerator correction efficiency tests) is a tracking code that is used to simulate transverse and longitudinal beam transport in linear accelerators. With this program it is possible to simulate the dynamic effects of a beam in the beam delivery system of the ILC, including the crab cavity. PLACET takes into account collective effects such as long and short range cavity wakefields, resistive and geometric wakefields in the collimators, and synchrotron radiation emission in all magnetic elements. Imperfections such as misalignments, phase and amplitude errors, as well as roll angles are also taken into account. In the beam delivery system, each bunch is split up into several macroparticles with each macroparticle representing thousands of electrons/ positrons.

The bunches were tracked to the IP using PLACET at which point a rotation of the axis by $7 \mathrm{mrad}$ was implemented into the electron positions mathematically using OCTAVE, hence creating a $14 \mathrm{mrad}$ crossing angle in the simulations.

The beams in the ILC and CLIC will be focused to very small sizes at the IP, hence the electromagnetic fields of each bunch will have a strong effect on each other causing bunches of opposite charge to attract each other. In the presence of bunch rotations or offsets, this beam-beam interaction leads to a mutual focusing which increases luminosity. However, if the beam-beam forces are too strong this can lead to instabilities which will decrease the luminosity. The beam-beam interaction was simulated using the code GUINEA-PIG (generator of unwanted interactions for numerical experiment analysis-program interfaced to GEANT). The phase space parameters of each macroparticle in the positron and electron bunches are

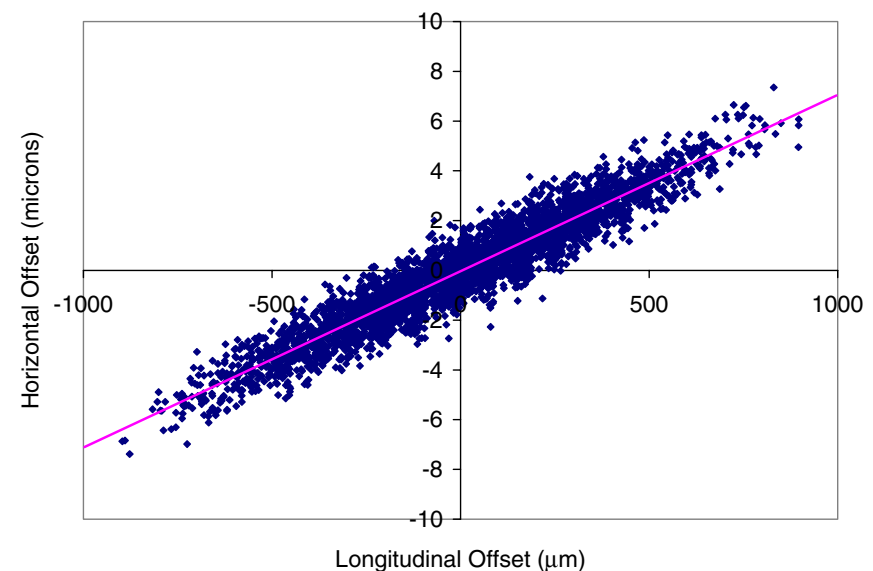

FIG. 3. (Color) The horizontal offset against longitudinal position for the macroparticles in a bunch at the IP of the ILC.

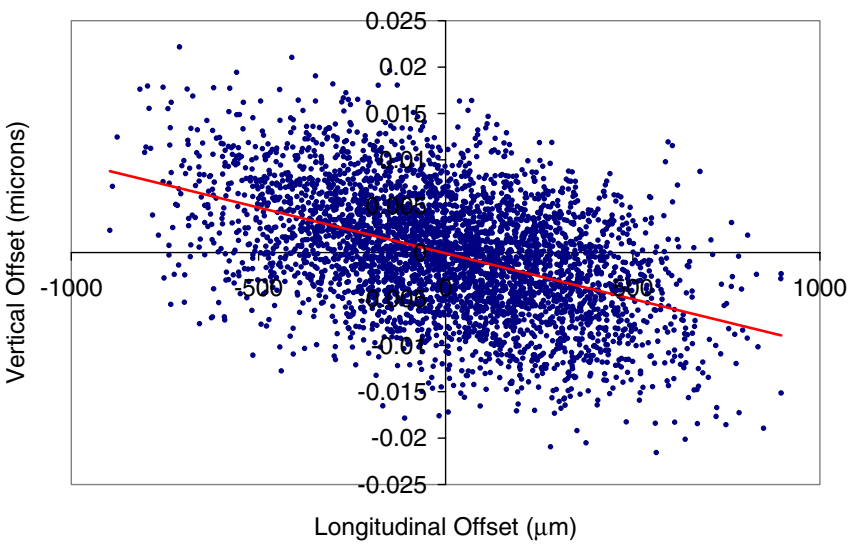

FIG. 4. (Color) The vertical offset against longitudinal position for the macroparticles in a bunch at the IP of the ILC where the crab cavity has a $10 \mathrm{mrad}$ roll misalignment.

passed to GUINEA-PIG to calculate the luminosity. In the simulations 80000 macroparticles per bunch were used. This number guarantees numerical stability to the GUINEAPIG calculations.

First, the effect of a roll misalignment on the crab cavity was calculated. A single 9 cell crab cavity was simulated at a voltage of $1.2936 \mathrm{MV}$ in order to achieve the $7 \mathrm{mrad}$ bunch rotation for a $250 \mathrm{GeV}$ beam. The horizontal rotation at the IP is shown in Fig. 3.

Then a rotational misalignment of $10 \mathrm{mrad}$ was used on one of the crab cavities and a rotation of the bunch in the $y-z$ plane was observed, shown in Fig. 4. This causes a vertical rotation of $17 \mu \mathrm{rad}$, which would cause a $30 \%$ luminosity loss with the ILC bunch size.

The bunch rotation was then systematically varied and the luminosity was recorded. In the simulations the cavity on one of the beam lines was rotated, and the luminosity was calculated as a function of misalignment, shown in Fig. 5. It was found that a roll alignment of $1.1 \mathrm{mrad}$ is

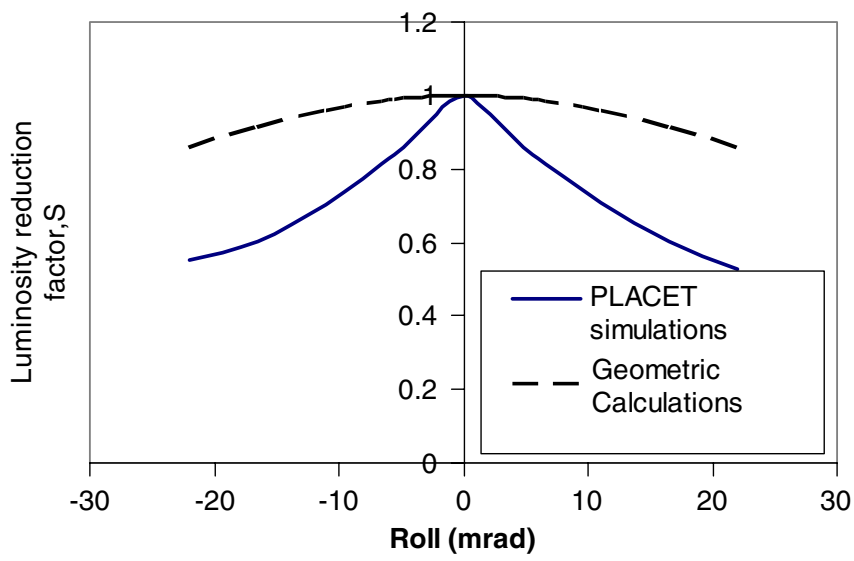

FIG. 5. (Color) Luminosity reduction factor for various roll misalignments of the ILC crab cavities comparing simulations and analytic calculations. 


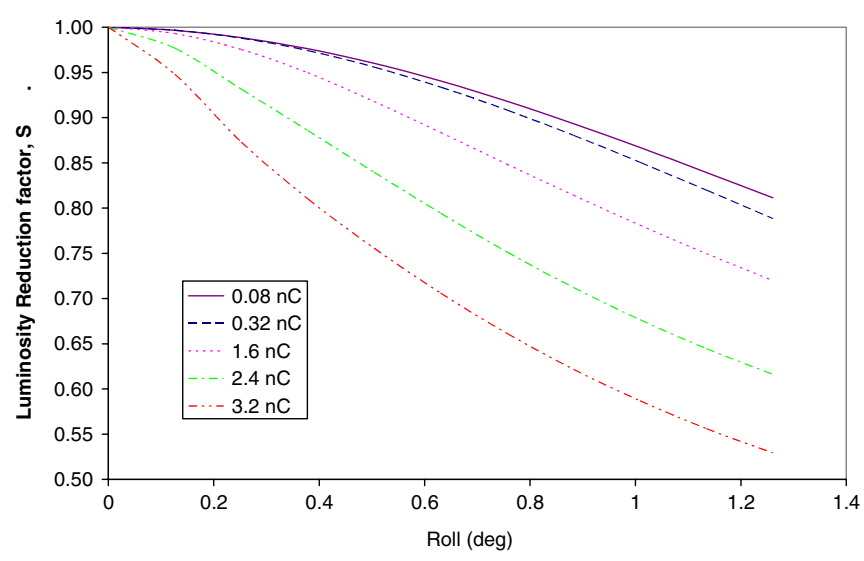

FIG. 6. (Color) Luminosity reduction factor for various roll misalignments of the ILC crab cavities as a function of bunch charge.

required to keep the luminosity loss below $2 \%$. The difference in the simulations compared to the geometric calculations is due to beam-beam effects where the force caused by the attraction of the opposite charges of electrons and positrons will disrupt the beam. The influence of beambeam effects was investigated by observing the luminosity as a function of cavity roll misalignment as a function of bunch charge, as shown in Fig. 6. It can be seen that as the bunch charge is reduced the calculated luminosity loss converges towards the analytical predictions. For example, for a $22 \mathrm{mrad}$ rotational misalignment the luminosity reduction factor varies from 0.81 at a bunch charge of $0.08 \mathrm{nC}$ to 0.53 at a bunch charge of $3.2 \mathrm{nC}$.

In order to correct for this effect an anticrab cavity with a deflecting voltage of $13 \mathrm{kV}$ was simulated in the beam delivery system next to the crab cavity. It was found that $100 \%$ of the luminosity was recovered and the rotation on the bunch was no longer visible, as can be seen in Fig. 7.

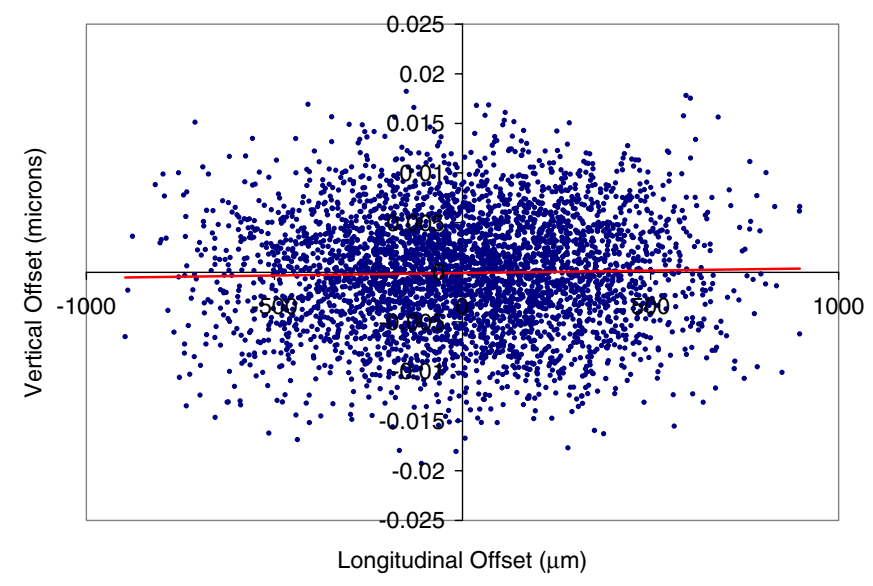

FIG. 7. (Color) The vertical offset against longitudinal position for the macroparticles in a bunch at the IP of the ILC where the crab cavity has a $10 \mathrm{mrad}$ roll misalignment and there is an anticrab cavity correction.

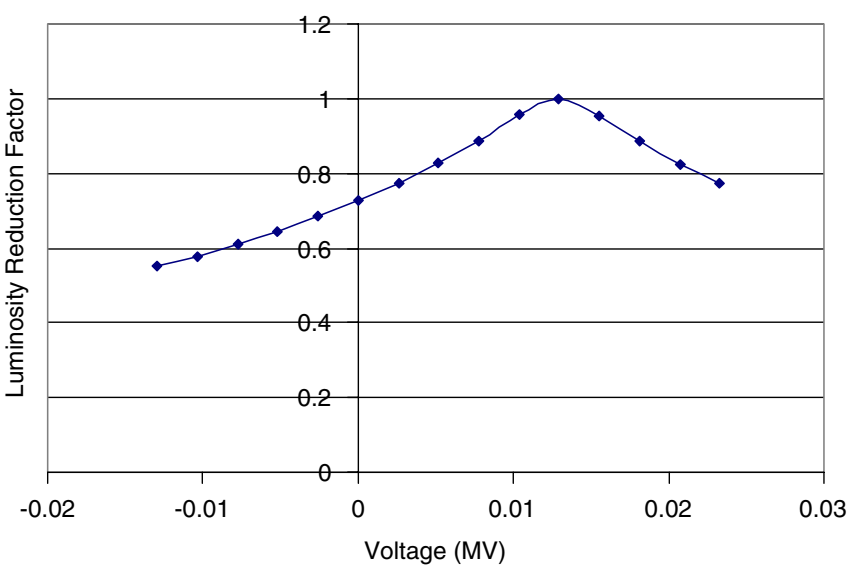

FIG. 8. (Color) Luminosity reduction factor as a function of the amplitude error on one anticrab cavity for a $10 \mathrm{mrad}$ roll misalignment of the ILC crab cavity.

Next the effect of phase and amplitude variations on the anticrab cavity was investigated. The cavities on both beam lines were misaligned by $10 \mathrm{mrad}$ and the luminosity of the collisions was calculated as the voltage of the anticrab cavity on one of the beam lines was varied between -0.013 and 0.013 MV. As can be seen in Fig. 8, a $20 \%$ variation in voltage results in a $5 \%$ luminosity reduction. This is unlikely to be an issue as modern accelerators tend to have amplitude stability of better than a few percent.

To investigate the effect on luminosity of phase variations in the anticrab cavity, the anticrab cavity voltage was set for maximum luminosity for a number of crab-cavity roll misalignments. Then the phase of one of the anticrab cavities was varied and the luminosity was calculated, shown in Fig. 9. With a roll misalignment of $100 \mathrm{mrad}$ a $0.1 \mathrm{deg}$ phase error, similar to the specifications for the ILC crab cavity, reduced the luminosity by $12 \%$. For a $22 \mathrm{mrad}$ misalignment a $0.1 \mathrm{deg}$ phase error resulted in a $1.5 \%$

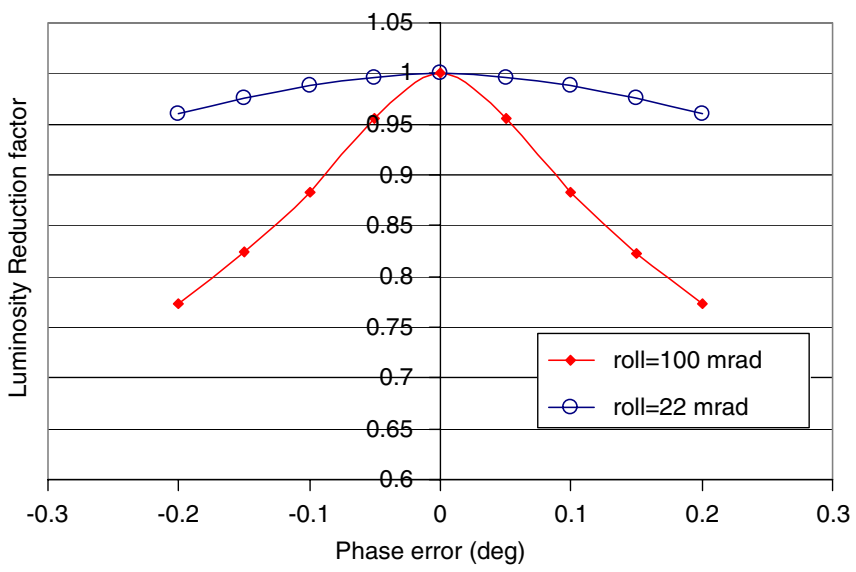

FIG. 9. (Color) Luminosity reduction factor as a function of the phase error on one anticrab cavity for various roll misalignments of the ILC crab cavity. 
luminosity loss. The difference with respect to the analytical results is due to beam-beam effects.

\section{CONCLUSION}

It has been shown in this paper that a misalignment of the crab cavities in an accelerator can produce an unwanted vertical crabbing/rotation of the beam. For the ILC crab cavities a $10 \mathrm{mrad}$ roll misalignment on one cavity can cause the loss of $30 \%$ of the luminosity.

One possible solution is to implement an anticrab cavity to remove the vertical momentum imparted to the particles. The operation of such a scheme has been simulated in PLACET and has been shown to recover $100 \%$ of the luminosity lost due to roll misalignments. Such a system has a limited range of correction, $\sim 30 \mathrm{mrad}$ for the ILC for a maximum $2 \%$ luminosity reduction due to phase errors, and would need to work in tandem with a mechanical tuning mechanism with a larger range but with less accuracy for the correction of roll misalignments. However, at $100 \mathrm{mrad}$ roll misalignment a luminosity reduction due to phase errors is only around $12 \%$ which is far less than the reduction in luminosity without any roll correction.

It is shown that for the beam parameters of the ILC the simple geometric approximations are not sufficient and full simulations, including beam-beam effects, are required.

The anticrab cavity need not be a superconducting if cavity; it could also be a simple normal conducting rf cavity such as the type used for beam diagnostics. This would keep the cost of such a tool low.

\section{ACKNOWLEDGMENTS}

The authors would like to thank Dr. Leo Bellantoni (FNAL), Mrs. Deepa Angal-Kalinin (STFC), Mr. Mike Dykes (STFC), and Dr. Andrei Seryei (SLAC) for their many useful discussions on this work.

[1] R. B. Palmer, Report No. SLAC-PUB-4707, 1988.

[2] T. Abe et al., in Proceedings of the 2007 Particle Accelerator Conference, Albuquerque, New Mexico, 2007 (IEEE, Albuquerque, New Mexico, 2007).

[3] C. Adolphsen et al., Report No. EUROTeV-Report-2007010.

[4] F. Zimmermann, in Proceedings of the 2007 Particle Accelerator Conference, Albuquerque, New Mexico, 2007, Ref. [2].

[5] J.P. Delahaye, in Proceedings of the 20th International Linac Conference, Monterey, CA, 2000 (SLAC Report No. MO201).

[6] D. Robin et al., Generation of Picosecond X-Ray Pulses in the ALS using RF Orbit Deflection, PAC 2005, Tennessee, in Proceedings of the Particle Accelerator Conference, Tennessee, 2005, pp. 3659-3661.

[7] A. T. d'Amico et al., in Proceedings of the Particle Accelerator Conference, Chicago, IL, 2001 (IEEE, New York, 2001).

[8] D. Schulte, in Proceedings of the Particle Accelerator Conference, New York, 1999 (IEEE, New York, 1999).

[9] B. Muratori, CERN Report No. AB-Note-2003-026.

[10] K. L. Brown, Report No. SLAC-PUB-0132, 1965.

[11] G. Burt, Report No. EUROTeV-Report-2006-098, 2006.

[12] G. Burt et al., IEEE Trans. Nucl. Sci. 54, 1728 (2007). 